Revue d'histoire de l'Amérique française

DE REVUE D'HISTOIRE DE L'AMÉRIQUE FRANÇAISE

\title{
Sur quelques effets de la rupture structurelle engendrée par la Conquête au Québec : 1760-1854
}

\section{Gérald Bernier}

Volume 35, numéro 1, juin 1981

URI : https://id.erudit.org/iderudit/303928ar

DOI : https://doi.org/10.7202/303928ar

Aller au sommaire du numéro

Éditeur(s)

Institut d'histoire de l'Amérique française

ISSN

0035-2357 (imprimé)

1492-1383 (numérique)

Découvrir la revue

Citer cet article

Bernier, G. (1981). Sur quelques effets de la rupture structurelle engendrée par la Conquête au Québec : 1760-1854. Revue d'histoire de l'Amérique française,

35(1), 69-95. https://doi.org/10.7202/303928ar d'utilisation que vous pouvez consulter en ligne. 


\title{
SUR QUELQUES EFFETS DE DE LA RUPTURE STRUCTURELLE ENGENDRÉE PAR LA CONQUÊTE AU QUÉBEC: 1760-1854*
}

\author{
GÉRALD BERNIER \\ Département de science politique \\ Université de Montréal
}

Il existe dans l'historiographie libérale de la Conquête un courant qui mise sur la "continuité historique»" ${ }^{1}$ et dont les conclusions sont reprises - dans un langage autre certes, mais substantiellement identique - par certains travaux d'inspiration marxiste ${ }^{2}$. Ce texte propose, au contraire, de voir la Conquête comme l'amorce d'une "rupture structurelle» dont les effets sont dans certains cas immédiats - tel l'impact sur les structures économiques et sociales - alors que dans d'autres - notamment le procès de transformation des rapports de production dans l'agriculture - ils s'étalent sur près d'un siècle d'histoire québécoise, soit jusqu'au début des années 1850 .

* Cette recherche a bénéficié de l'appui financier du ministère de l'Éducation du Québec dans le cadre du programme Formation de Chercheurs et Action concertée (FCAC). L'auteur tient également à remercier les assistants de recherche Danielle Laudy, Daniel Salée et Vinh Ta Van dont les rapports préliminaires de recherche ont été un précieux atout pour la rédaction de ce texte. Mon collègue Gérard Boismenu a commenté une première version du texte, je l'en remercie.

1 On pense ici plus particulièrement à certains travaux des représentants de «l'école de Québec». Voir Fernand Ouellet, Jean Hamelin, Marcel Trudel, etc. Au Canada anglais, les travaux d'Innis et de Creighton s'inscrivent dans cette ligne de pensée.

2 Gilles Bourque, Classes sociales et question nationale au Québec, 1760-1840 (Montréal, Parti Pris, 1970); Gilles Bourques et Nicole Frenette, «La structure nationale québécoise», Socialisme Québécois, nos 21-22 (avril 1971): 109-156. Tout le raisonnement de l'auteur se fonde sur le postulat d'une Nouvelle-France vivant à l'heure du mode de production capitaliste (voir Classes sociales..., 45 ss). D'où la formulation d'énoncés «continuistes" tels que:

«Mais dans le cas du changement de régime qui s'est effectué au Québec en 1760, seules les caractéristiques nationales de la formation sociale sont atteintes». Ou encore: «Toute la difficulté de fournir une explication adéquate de la Conquête réside dans le fait qu'elle semble précisément sur le plan structurel, ne provoquer aucune rupture: seul le nom de cette formation sociale semble avoir changé» («La structure nationale...», 126-127).

Dans un ouvrage plus récent, l'auteur porte plus d'attention aux problèmes de l'articulation des modes de production et du niveau de développement du capitalisme en métropole. Cependant, en raison de la brièveté de la démonstration, les raisonnements ne sont qu'esquissés. Voir Gilles Bourque et Anne Legaré, Le Québec. La question nationale (Paris, Maspero, 1979), chapitre 2. Plus particulièrement, voir les pages 24-25. 
Dans l'immédiat, au sein d'une société où prédominait encore au niveau des rapports de production le mode de production féodal (MPF), une structure reflétant le capitalisme le plus avancé de l'époque est brutalement introduite de l'extérieur. De sorte que certaines institutions et pratiques héritées du régime français et, en apparence, maintenues intactes sous le régime anglais, changent de sens (signification) et de fonction après la Conquête. L'étude de la période 1760-1854, tout particulièrement dans le secteur agricole, permet de saisir dans le plus long terme la rupture qui s'est opérée en dépit des apparences formelles de continuité. Cette rupture consiste en une inversion de la matrice de dominance du procès d'articulation des modes de production rencontrée sous la NouvelleFrance. De dominé qu'il était avant la Conquête, le mode de production capitaliste (MPC) devient dominant sans que l'impulsion première en faveur de ce changement ait été générée de l'intérieur. En bref, la Conquête abrège singulièrement la durée de la transition d'une économie dominée par un procès et des rapports de production de type féodal à une économie capitaliste ${ }^{3}$. En un temps relativement court, par suite de la Conquête, la morphologie de la structure de classes se transforme radicalement, de même que l'indice hégémonique dans la composition du bloc au pouvoir.

Ce renversement de la matrice de dominance tient en bonne partie, selon nous, à l'état inégal du développement du MPC au sein des métropoles (France et Angleterre) au moment de leur pénétration coloniale respective et aussi au moment où survient la Conquête. En d'autres termes, d'une part l'Angleterre de 1760 est beaucoup plus proche de la phase industrielle du MPC que la

3 La réflexion proposée s'alimente à divers travaux théoriques portant sur la problématique de la transition et de l'articulation des modes de production. Parmi les ouvrages consultés, on retrouve ceux de Barry Hindess et Paul Q. Hirst, Pre-capitalist modes of production (Londres, Routledge et Kegan Paul, 1975); Mode of production and social formation (Londres, MacMillan, 1977); CEREM, Sur les sociétés précapitalistes (Paris, Editions Sociales, 1978); Maurice Dobb et Paul M. Sweezy, Du féodalisme au capitalisme: problèmes de la transition (Paris, Petite collection Maspero, 2 tomes, 1978); Louis Althusser et Étienne Balibar, Lire le capital, Tome II (Paris, Petite collection Maspero, 1975): Perry Anderson, L'État absolutiste, Tome $1-L$ 'Europe de l'ouest (Paris, Maspero, 1978); Paul Boccara, Sur la mise en mouvement du «capital» (Paris, Editions Sociales, 1978); PierrePhilippe Rey, Les alliances de classes (Paris, Maspero, 1973); Guy Dhoquois, Pour l'histoire (Paris, Anthropos, 1971).

Le concept d'articulation est employé dans le sens que lui confère Pierre-Philippe Rey, c'est-à-dire pour caractériser les divers rapports entre modes de production différents. Cet emploi s'éloigne du sens donné au même concept par Louis Althusser. Pour Althusser l'articulation réfère aux rapports entre les diverses instances (ou structures régionales) d'un même mode de production.

Selon le contexte, le concept de transition peut désigner soit le passage d'un mode de production à un autre (transition inter-modes de production), soit le passage d'une phase de développement à une autre à l'intérieur d'un même mode de production (transition intramode de production; par exemple, le passage de phase marchande à la phase industrielle dans le cours du développement du MPC). Voir Boccara, op. cit., 73 ss. 
France de 1608 et, d'autre part, cette Angleterre de 1760 achève plus que la France de la même époque de réunir les conditions nécessaires au passage à la phase industrielle ${ }^{4}$. Cet état de fait est appelé à se refléter sur les instances structurelles que l'on retrouve dans la colonie sous chacun des régimes. Dans la mesure, en effet, où toute entreprise coloniale tend à reproduire une partie des structures métropolitaines, les structures sociales de la NouvelleFrance refléteront la période de transition qui caractérise l'économie française durant les $17^{\mathrm{e}}$ et $18^{\mathrm{e}}$ siècles, marquant le passage du féodalisme au capitalisme. La noblesse, l'Église, le régime seigneurial avec ses seigneurs et censitaires, voilà autant de traits caractéristiques du féodalisme qui imprègnent les structures sociales coloniales sous le régime français. En outre, l'état peu avancé du développement du MPC en France, notamment en ce qui concerne le règlement de la question paysanne, explique les difficultés de peuplement que connaîtra la Nouvelle-France durant toute la durée du régime français.

À l'opposé, l'Angleterre de 1760 «exporte» des éléments structuraux qui témoignent de sa plus grande proximité du MPC de la phase industrielle. La structure économique de la colonie deviendra rapidement plus complexe. La classe capitaliste d'abord confinée aux marchands se complétera, après 1846, de financiers et d'industriels. La population croîtra rapidement par suite du déversement par l'Angleterre de ses surplus de main-d'oeuvre.

Cette prise en considération de l'état du développement des métropoles est fondamentale pour notre propos. En effet, la diver-

4 Nous n'avons pas à nous intéresser de manière détaillée à l'histoire économique des métropoles, non plus qu'à spéculer sur ce qu'il serait advenu du régime français par suite de la Révolution de 1789 dans l'hypothèse où la Conquête ne serait pas survenue.

Il existe une littérature très développée sur l'histoire économique respective de l'Angleterre et de la France, de même que sur leur histoire économique comparée. On y retrouve un certain nombre de controverses et de divergences d'interprétation mais, en général, les auteurs s'entendent pour dire que l'Angleterre est le premier pays à réunir les conditions qui favorisent l'apparition du capitalisme de la phase industrielle et pour situer les débuts de la révolution industrielle en Angleterre à peu près à l'époque où survient la Conquête. On insiste beaucoup sur le règlement hâtif de la question paysanne à cet égard, c'est-à-dire la révolution agricole et l'expropriation de la paysannerie. En France, par ailleurs, la Révolution retardera le procès d'industrialisation en émiettant la propriété foncière au lieu de la concentrer comme ce fut le cas en Angleterre avec les enclosures.

Pour plus de détails voir au sujet de l'Angleterre les travaux de T.S. Ashton, $L a$ révolution industrielle, 1760-1830 (Paris, Plon, 1955). (L'introduction de Claude Fohlen à cet ouvrage compare l'état de développement de la France pour la même période); Christopher Hill, Reformation to industrial revolution (Harmondsworth, Penguin Books, 1969); J.H. Plumb, England in the eighteenth century (1714-1815) (Harmondsworth, Penguin Books, 1969); on trouvera également de nombreuses données dans les travaux de Eric J. Hobsbawn. Sur la France, consulter les travaux de François Crouzet, M. Lévy-Leboyer, Claude Fohlen, A.-L. Dunham. Parmi les travaux plus généraux mentionnons l'ouvrage de Maurice Dobb, Études sur le développement du capitalisme (Paris, Maspero, 1971). On trouvera des éléments plus proprement comparatifs chez Crouzet, Lévy-Leboyer et Fohlen. 
gence de l'interprétation proposée par rapport aux conclusions formulées par une certaine historiographie libérale au sujet de l'impact de la Conquête tient précisément à la caractérisation qui est faite de la Nouvelle-France. Pour cette dernière et ses sympathisants néo-marxistes, la conception de la Conquête en tant que "continuité historique» repose sur le postulat voulant que la Nouvelle-France ait connu une forme de développement capitaliste par son commerce de fourrures. Cette conception conduit, par conséquent, à caractériser l'ensemble de la formation NouvelleFrance comme étant dominé par le MPC. Cette vue des choses, erronée selon nous, rend alors superflu tout recours à la notion de transition et à celle de l'articulation de différents modes de production. Au surplus, elle conduit les néo-marxistes à la formulation d'une fausse problématique, celle d'une «double structure de classes» qui résulterait directement de la Conquête ${ }^{5}$.

\section{A - Une problématique de la transition appliquée à la Conquête}

En acceptant de caractériser la formation Nouvelle-France comme étant dominée par des rapports de production féodaux, la tâche du chercheur est tout autre. L'analyse projette au premier plan des concepts tels ceux de rapports de production, relations de propriété, forces productives, classes sociales, etc. Son foyer central repose sur l'articulation des différents modes et formes de production. Toute la problématique de la Conquête est alors appréhendée dans ces termes.

L'ambiguïté des interprétations évoquées plus haut tient, entre autres, à la façon de disposer de la «bourgeoisie marchande» de la Nouvelle-France. L'existence d'une telle bourgeoisie ne signifie pas nécessairement la dominance du MPC ainsi qu'en sont amenés à conclure ces historiens. Car, à ce compte, s'il fallait conclure à la dominance du MPC à chaque fois que l'on rencontre une soi-disant «bourgeoisie marchande» dans le cadre d'une société, il faudrait qualifier de «capitalistes» certaines formations antiques et médiévales. Les questions de théorisation se posent donc avec acuité; il faut posséder une théorie du capital marchand et de son articulation à un mode de production donné. Ainsi, on pourra dis-

5 Gilles Bourque, op. cit.; Denis Monière, «L'utilité du concept de mode de production des petits producteurs pour l'historiographie de la Nouvelle-France», Revue d'histoire de l'Amérique française, 29, 4 (mars 1976): 483-502.

Pour une discussion des égarements théoriques que recèle cette problématique, voir Gérald Bernier, «La structure de classes québécoise au $19 \mathrm{e}$ siècle et le problème de l'articulation des modes de production", à paraître, Revue canadienne de science politique.

6 Cette partie reprend certains des énoncés contenus dans le rapport de recherche de Vinh Ta Van, "La Conquête de 1760. Esquisses pour une recherche», Département de science politique, automne 1979, $45 \mathrm{p}$. 
tinguer la phase marchande du MPC d'avec l'activité marchande que l'on rencontre dans des contextes où dominent d'autres modes de production? ${ }^{7}$. Car l'échange marchand ne constitue pas forcément le stade préliminaire qui conduit au MPC achevé.

Pour savoir si une activité marchande est du type qui constitue une phase préliminaire du MPC achevé ou si, au contraire, elle s'insère comme appendice dominé dans le cadre de la domination d'un autre mode de production, il faut procéder à l'analyse de l'ensemble des rapports de production dans une formation sociale donnée dans le but d'y dégager la place et la fonction de cette activité.

Les contraintes d'espace empêchent toute analyse exhaustive de la structure économique de la Nouvelle-France. En bref, la colonisation de la Nouvelle-France doit être comprise en fonction de la logique de la monarchie absolue française puisqu'elle est issue des politiques de ce système. Il s'agit d'un système de transition marquant les débuts de la phase de l'accumulation du capital en France. Le féodalisme n'arrive plus à se reproduire intégralement; la noblesse foncière est sur son déclin. Or, le commerce extérieur servira à financer la Couronne en remplacement des revenus que lui assuraient jusque-là les seigneurs ${ }^{8}$. Dans cette optique, le commerce, notamment celui des fourrures, se veut la raison première de l'établissement de la colonie. Il doit commander tout le reste, y compris l'infrastructure agricole devant servir de soutien à cette activité marchande.

Les conditions existantes dans la colonie, fort différentes de celles prévalant dans la métropole ${ }^{9}$, font que durant tout le régime français, le commerce conservera toujours un caractère d'extériorité par rapport à la colonie. De telle sorte que l'on peut parler d'une structure à deux étages. Sur le plan strictement économique, l'échange propre à la phase marchande du MPC caractérise l'acti-

7 Fossaert fait allusion à ce genre de problème à propos des marchands dans les cités italiennes du Moyen-Âge. On peut raisonner de la même façon en ce qui concerne la Nouvelle-France.

«Quelques rarissimes marchands, qui délaissent l'artisanat, se spécialisent dans le commerce et se font même un peu banquiers, ne suffisent pas à former une classe capitaliste marchande, dans les jeunes cités italiennes: il faut en outre, que leur nombre s'accroisse, que leur fonction se stabilise, que les liens se multiplient entre eux et leurs homologues des cités voisines ou leurs agents des foires lointaines, pour que les singularités de leurs positions et de leurs liens personnels s'effacent derrière une loi commune qui devient la loi de leur fonction, de leur classe, et qui devient aussi l'aune à laquelle leurs comportements singuliers se mesurent comme écarts» (Claude Fossaert, La Société. Tome 4 - Les classes (Paris, Seuil, 1980), 38).

8 Voir à ce sujet L. R. Macdonald, «France and New France: the internal contradictions», Canadian Historical Review, 52, 2 (juin 1971): 121-143.

9 Pour une analyse de ces différences, voir Louise Dechêne, Habitants et marchands de Montréal au XVIIe siècle (Montréal, Plon, 1974). 
vité qui lie la colonie à la métropole, notamment par le commerce des fourrures. Par ailleurs, les activités économiques internes de la colonie, qu'il s'agisse du secteur agricole ou de celui de l'artisanat et de la petite production marchande constituent des formes de production des biens matériels non-capitalistes. Le régime seigneurial s'apparente au MPF, tandis que l'artisanat et la petite production relèvent de formes de production marchande simple. Quant aux rapports de production, en raison de la prépondérance de la tenure seigneuriale au niveau des structures agraires ainsi que de l'importance des effectifs mobilisés par l'agriculture, on peut dire que la Nouvelle-France est, dans l'ensemble, dominée par des rapports de production apparentés au MPF ${ }^{10}$.

Le caractère non-capitaliste de la Nouvelle-France se vérifie en outre par la place qu'y tiennent les instances non-économiques dans la reproduction des rapports de production. Le producteur n'a pas encore été séparé de ses moyens de production. Par conséquent, l'appropriation du surproduit par le seigneur n'est pas directement inscrite dans le procès de production même. Elle s'appuie sur le droit (relevant de l'instance juridico-politique) qui définit la nature des rapports économiques entre censitaire et seigneur ${ }^{11}$.

S'il est ici question de féodalisme, il faut préciser qu'il s'agit d'un féodalisme «bâtardisé», en voie de désintégration, puisque tel que souligné antérieurement, la transition vers le capitalisme est déjà amorcée dans la métropole au moment de la pénétration coloniale française et qu'elle s'accentuera pendant la durée de la présence française au Canada. Par conséquent, la colonisation en tant qu'excroissance de la métropole est appelée à réfléchir ces traits.

10 À cet égard, Maurice Séguin souligne le peu d'importance de l'agriculture dans les rapports d'échange entre la métropole et la colonie. Même le marché intérieur était de faible dimension. De sorte que l'agriculture qui se pratiquait en était une de subsistance. Elle accaparait sous le régime français les trois quarts de la population. Ces données renforcent l'idée selon laquelle le commerce conserva un caractère d'extériorité par rapport à la colonie durant tout le régime français (La Nation «canadienne» et l'agriculture (1760-1850) (Trois-Rivières, Boréal Express, 1970), 49).

11 Pour plus de détails, voir Althusser et Balibar, op. cit., 103 ss. Dans ce texte, Balibar montre que dans le cas du MPF le surproduit n'appartient pas de lui-même aux représentants de la classe dominante puisque le serf possède ses moyens de production ce qui lui permet d'assurer sa propre subsistance. Et ce, contrairement au MPC où l'ouvrier est dépendant d'un salaire pour subsister. Alors que dans le MPC, le capitaliste s'approprie directement le surtravail, dans le MPF, cette appropriation passe par certaines prescriptions dictées par l'instance juridico-politique et ne se fonde pas directement sur le rapport économique seigneur-censitaire. Bref, pour que le seigneur puisse s'approprier le surtravail des serfs, l'existence explicite d'un rapport politique devient nécessaire, ce rapport prenant soit «la forme de la violence «pure», soit les formes aménagées et améliorées du droit» (p. 103). Voir également Nicos Poulantzas, Les classes sociales dans le capitalisme d'aujourd'hui (Paris, Seuil, 1974), 14-17. 
Néanmoins, dans le cadre de la Nouvelle-France, l'activité marchande se déroule dans un contexte qui est largement dominé par des rapports de production féodaux, de sorte que la «bourgeoisie» marchande que l'on y rencontre est une bourgeoisie marchande féodale. La bourgeoisie marchande de la Nouvelle-France n'est pas, en effet, du type de celle qui annonce la mise en place des structures nécessaires au MPC achevé. Rien dans son activité conduit à l'émergence des ingrédients nécessaires au développement du MPC, à savoir la transformation de la richesse d'argent en capital (accumulation) et la séparation du travailleur de ses moyens de production (constitution d'un réservoir de main-d'oeuvre) qu'occasionnent les divers mécanismes d'expulsion ou de dépossession de la paysannerie ${ }^{12}$. Or rien de cela n'est présent en NouvelleFrance ou susceptible de l'être dans un avenir rapproché ${ }^{13}$. La classe de marchands coloniaux est de la même famille que la «bourgeoisie» féodale française décrite par Porshnev et Althusser ${ }^{4}$. L'activité commerciale de la Nouvelle-France est conduite en marge de l'agriculture et essentiellement orientée vers la métropole, éléments très importants pour la compréhension du procès d'articulation des modes de production durant le régime français.

La politique coloniale de la monarchie absolue française avait cherché à "épurer» le régime seigneurial canadien des contradictions et conditions d'exploitation aiguës qui, en France, avaient contribué à amorcer la désintégration du féodalisme. C'est dans cette optique qu'il faut voir les arrêts de Marly de 1711, freinant la cupidité naturelle des seigneurs. Cependant, malgré ces «adoucissements» en regard des conditions de vie du censitaire, on observe, à la fin du régime français, une accentuation du caractère féodal

12 Les marchands de la Nouvelle-France ne pouvaient rencontrer les exigences de l'accumulation tant que se maintiendrait le lien colonial. Jean Hamelin souligne, en effet, qu'entre 1675 et $1760,72 \%$ du revenu produit par le commerce des fourrures fut retourné en France. La base d'accumulation pour les marchands locaux était donc assez mince (Economie et société en Nouvelle-France (Québec, PUL), 55-56).

13 Voir à ce sujet les conclusions de L.R. Macdonald, op. cit., 130-143.

14 Boris Porshnev, Les soulèvements populaires en France au XVIIe siècle (Paris, Flammarion, coll. Champs, 1972).

Au sujet du $18 \mathrm{e}$ siècle français, Louis Althusser écrit: «La véritable bourgeoisie moderne... est la bourgeoisie industrielle... Or cette bourgeoisie était dans sa généralité inconnue du XVIIIe siècle. La bourgeoisie de cette période était toute différente: elle reposait essentiellement dans ses éléments les plus avancés sur l'économie marchande. De ce que l'économie industrielle est sortie, à un moment donné, de l'accumulation dont l'économie marchande constitue un moment, on tire trop souvent la conclusion que l'économie marchande [et la «bourgeoisie» qui s'y rattache] était dans son principe étrangère à la société féodale. Rien n'est plus douteux. Il suffit en effet de voir dans quel sens jouait alors cette économie marchande pour conclure qu'elle était une pièce assez bien intégrée du système féodal lui-même: le mercantilisme est justement la politique et la théorie de cette intégration" (Louis Althusser, Montesquieu, la politique et l'histoire (Paris, PUF, 1974), 113-114. Les soulignés et la parenthèse sont de nous). 
du régime seigneurial par rapport à ce qu'il était durant les premières décennies de son implantation. Dans les seigneuries les mieux situées et là où le taux d'occupation est très élevé, on n'hésite pas à mettre en vigueur de nouveaux droits et servitudes que permet le droit coutumier régissant la tenure seigneuriale ${ }^{15}$. Une telle situation rapproche des conditions d'exploitation de la seigneurie en France et aurait, tôt ou tard, produit les mêmes effets en NouvelleFrance, soit une accumulation de contradictions conduisant à la désintégration des rapports de production féodaux.

La Conquête s'en chargera puisqu'avec elle sont levées les lois restrictives qui protégeaient le censitaire d'une exploitation plus accentuée du seigneur. S'amorce alors pour la formation canadienne la phase de transition du féodalisme au capitalisme puisque sont déclenchés, dans la logique même du capitalisme anglais, le procès d'expropriation et de prolétarisation de la paysannerie et le renversement du procès d'articulation des modes de production avec l'instauration du MPC comme matrice dominante ${ }^{16}$. La colonisation anglaise issue d'une métropole plus avancée va lever le blocage de l'élargissement de la propriété foncière en abolissant, dans les faits, les lois restrictives françaises. Car le renforcement du caractère féodal du régime seigneurial, durant le premier deuxtiers de siècle qui suit la Conquête, attesté par la décélération du rythme des concessions après 1820, par la multiplication des réserves dans les contrats, par l'augmentation des taux de cens et de rentes pour les nouvelles concessions, par le recours à la pratique du titre nouvel, par la remise en force de droits tombés en désuétude, etc., $n$ 'est qu'apparent ${ }^{17}$. Sa signification ne répond plus à la

15 Louise Dechêne, «L'évolution du régime seigneurial au Canada. Le cas de Montréal aux XVIIe et XVIIIe siècles», Recherches sociographiques, XII, 2 (mai-août 1971): 143-184.

16 Le sens à donner au concept d'expropriation de la paysannerie tel qu'utilisé dans ce texte renvoie à la finalité du procès plutôt qu'aux modalités. La fin visée est la création d'un surplus de main-d'oeuvre. Les moyens pour y parvenir sont nombreux. Ils varient suivant les contextes historiques. L'Angleterre, par exemple, recourt à l'expropriation au sens strict (Voir Maurice Dobb, Études sur le développement du capitalisme (Paris, Maspero, 1971), $240 \mathrm{ss}$ ). Au Canada, diverses mesures visant à rendre difficile l'accès à la propriété foncière se conjuguent pour produire ce surplus de main-d'oeuvre. Pour dissiper l'équivoque, nous emploierons les termes «exclusion de l'accès à la propriété foncière», «dépossession» pour qualifier ce procès dans le cas canadien.

17 «Le titre nouvel est une pratique légale consistant à obliger les paysans à prendre un nouveau titre de propriété dans lequel le seigneur fixe les conditions lui-même. C'est un acte notarié rédigé par un notaire par lequel le paysan s'engage à payer les arrérages dus sur sa terre et à payer le nouveau taux des rentes fixé par le seigneur" (Lise Pilon-Le, "Le régime seigneurial au Québec: contribution à une analyse de la transition au capitalisme», Les Cahiers du socialisme, no 6 (automne 1980): 165).

Cette pratique semble avoir été assez répandue durant les années 1820 (155-156) permettant ainsi aux seigneurs de circonscrire la fixité des redevances prescrites par le droit français. De sorte que les hausses de taux de cens et de rentes portent non seulement sur les nouvelles concessions mais atteignent également, par cette pratique, les anciennes seigneuries. 
logique de la surexploitation féodale, mais bien à celle capitaliste de la nécessité de constituer, à plus ou moins long terme, une base d'accumulation de capital et une main-d'oeuvre «nue et libre».

Cette perspective de la rupture échappe totalement à un courant d'interprétation qui se laisse prendre au piège des apparences de la continuité. On nous signale, en effet, que malgré la Conquête, les structures socio-économiques continuèrent d'être mercantiles et seigneuriales, demeurant, encore pendant un demisiècle, axées sur le commerce des fourrures; que la tenure seigneuriale fut maintenue jusqu'en 1854 et que, finalement, la puissance de l'Église fut rétablie et renforcée. Fernand Ouellet, entre autres, s'avère impuissant à rendre compte du changement d'attitude des commerçants anglais à l'égard du régime seigneurial. Eux qui, dans un premier temps, font l'acquisition de seigneuries puis, dans un second, réclament l'abolition de ce système de tenure. Pour Ouellet, ce revirement s'explique par le fait qu'initialement «le prestige l'emporta sur les considérations d'intérêts économiques» alors que ces dernières «deviendront plus pressantes par la suite» ${ }^{18}$. On conviendra aisément que cette «explication» n'en n'est pas une et que l'auteur est mystifié par son objet d'analyse. Car elle revient à dire que, pendant un temps, les seigneurs-commerçants anglais adoptèrent un comportement irrationnel et que, par magie ou par lassitude (on ne peut pas jouer aux aristos toute sa vie, quand même!), la raison reprit le dessus.

Pierre Tousignant fournit indirectement des assises plus solides à l'explication de ce comportement des marchands anglocanadiens en portant l'analyse en terrain métropolitain ${ }^{19}$. Son étude de la genèse de l'Acte constitutionnel de 1791 présente une Angleterre qui, malgré les immenses progrès réalisés au plan économique par les forces capitalistes confère toujours à la propriété foncière une place de premier choix aux plans politique et idéologique. La propriété foncière demeure l'assise du pouvoir politique et l'idéologie dominante véhicule les valeurs de l'aristocratie foncière. Il faudra attendre les grandes réformes des années 1830 pour que le pouvoir passe aux mains de la bourgeoisie.

Cette situation expliquerait, entre autres, l'attrait qu'exerce sur les marchands britanniques installés au Canada, durant les premières décennies qui suivent la Conquête, la propriété foncière et

18 «Le régime seigneurial dans le Québec, 1760-1854», in Claude Galarneau et Elzéar Lavoie (sous la direction de), France et Canada français du XVIe au XXe siècle (Québec, PUL, 1966), 167.

19 Pierre Tousignant, «Problématique pour une nouvelle approche de la constitution de 1791", Revue d'histoire de l'Amérique française, 27, 2 (septembre 1973): 181-234. 
le domaine seigneurial ${ }^{20}$. D'autant plus que par la libéralité du droit de suffrage accordé en 1791 - laquelle, on le sait, jouera en faveur des représentants issus de la petite bourgeoisie des professions libérales - ces mêmes marchands ont été écartés du contrôle qu'ils entendaient exercer sur la branche législative élective. Par l'Acte de 1791, la Grande-Bretagne a cherché à reproduire le modèle métropolitain qui assure aux intérêts issus de la propriété foncière le contrôle effectif du pouvoir. Les rapports de pouvoir engendrés par la Constitution de 1791 ont donc contraint les marchands à effectuer un rapprochement avec les représentants de l'administration - souvent issus de l'aristocratie foncière britannique ou du moins représentant ses intérêts - de même qu'avec les éléments aristo-cléricaux (seigneurs et Église) canadiens-français. Ensemble, ils se partageront les postes au sein du Conseil exécutif et du Conseil législatif. Pour les marchands, il s'agit d'une volteface par rapport à leur comportement politique de la période 17601791 puisqu'ils avaient alors lutté, en liaison avec certains éléments réformistes canadiens (français), pour l'obtention d'une Chambre d'assemblée sans se douter des résultats qui iraient finalement à l'encontre de leurs intérêts ${ }^{21}$. L'alliance un peu contre-nature contractée par suite de la conjoncture issue de 1791 forcera la bourgeoisie marchande «à se retrancher derrière un conservatisme politique» qui se maintiendra jusqu'aux années $1840^{22}$.

De fait, toute cette question de l'attitude de la bourgeoisie marchande à l'égard de la propriété foncière s'éclaire lorsqu'envisagée dans le cadre d'une problématique de la transition du féodalisme au capitalisme. Pierre-Philippe Rey dégage trois phases dans ce procès de transition: 1 ) renforcement par le capitalisme des rapports de production et d'exploitation précapitalistes; 2 ) le capitalisme se subordonne les modes de production précapitalistes tout en continuant à les utiliser; 3) le capitalisme fait disparaître ces

20 Une raison non moins fondamentale est le fait qu'au fur et à mesure où l'économie coloniale se développe l'achat d'une seigneurie s'avère une bonne affaire. On peut tirer de son exploitation des revenus intéressants, substantiellement plus élevés que durant le régime français, comme l'a démontré Fernand Ouellet dans des travaux récents. Voir «Propriété seigneuriale et groupes sociaux dans la vallée du Saint-Laurent (1663-1840), in Cahiers du centre de recherche en civilisation canadienne-française, Mélanges d'histoire du Canada français offerts au professeur Marcel Trudel (Ottawa, Éditions de l'Université d'Ottawa, 1978), 183-213; «Libéré ou exploité! Le paysan québécois d'avant 1850», Histoire Sociale, XIII, 26 (novembre 1980): 339-368.

21 Pierre Tousignant, op. cit., 186-187.

22 Ibid., 184. 
autres modes de production ${ }^{23}$. On devra y revenir plus en détail. Pour l'instant, qu'il suffise de souligner que le revirement d'attitude (noté par Ouellet) chez les seigneurs-commerçants s'inscrit tout à fait dans la logique du procès de transition. C'est-à-dire que si, dans un premier temps, le capitalisme naissant maintient la propriété foncière de type féodal et, en apparence, en renforce certains traits (en contribuant, par exemple, à l'accroissement du niveau d'exploitation des censitaires), il arrive, dans un second temps, que cette forme de propriété, par suite d'une évolution des forces productives et des rapports de production, devienne un obstacle à l'épanouissement du capitalisme, obstacle qu'il faut alors abattre. C'est précisément ce qui se produit avec le régime seigneurial canadien comme on sera à même de le constater plus loin.

Ceux qui abordent la Conquête dans la perspective de la «continuité historique» sont en quelque sorte victimes d'une illusion d'optique obtenue sous l'effet de l'assimilation par analogie de deux structures dissemblables à partir de ressemblances entre leurs éléments respectifs. Ce faisant, on abstrait les parties de l'ensemble que l'on compare entre elles et, trouvant certaines ressemblances, on conclut alors que les ensembles sont identiques. Il y a là erreur de logique.

Ce qu'il faut plutôt saisir, c'est qu'avec la venue de l'Angleterre - soit un ensemble de structures où le MPC est dominant les structures socio-économiques canadiennes, en apparence res-

23 Pierre-Philippe Rey, Les alliances de classes (Paris, Maspero), 74 ss.

«Tant que la bourgeoisie a besoin des modes de production antérieurs et des rapports de production qui les caractérisent, elle ne peut que maintenir en vie ces modes de production, renforcer ces rapports d'exploitation, s'allier avec les classes dominantes des anciens modes de production. C'est précisément ce qui se passe pendant les premières étapes du procès de domination capitaliste» (16). Tandis que durant la troisième phase:

«La présence des propriétaires fonciers n'est plus nécessaire au capital... ni pour son approvisionnement en forces de travail (qui est assuré par la ruine des petits paysans et petits fermiers) ni pour son approvisionnement en matières premières agricoles, ni pour l'approvisionnement vivrier des ouvriers et des capitalistes eux-mêmes, qui sont fournis aux marchés urbains par les producteurs agricoles capitalistes» (76).

Bien entendu, les modalités de la dissolution, de même que le temps requis pour y parvenir, varient grandement d'une situation à l'autre. De même, tout procès de transition se confronte à deux tendances contraires. L'une tend à la dissolution, l'autre à la conservation. Lorsque l'on dit qu'un mode de production disparaît, il faut comprendre qu'il disparaît en tant que mode de production distinct, en ce qu'il n'arrive plus à se produire. Peuvent néanmoins subsister des formes de production issues de ce mode de production dissous. C'est notamment le cas, pour ne prendre qu'un exemple, de la petite production agricole familiale sous le capitalisme avancé. Voir Alain Lipietz, Le Capital et son espace (Paris, Maspero, 1977), en particulier, 33-53. 
tées les mêmes, vont complètement changer de sens ${ }^{24}$. Intégrées de force dans un ensemble de structures (l'Angleterre) régi par des rapports de production et des rapports sociaux capitalistes, elles tendent, cette fois, à reproduire, dans un mouvement élargi, ces rapports capitalistes et l'accumulation capitaliste, même si elles rencontrent ou engendrent forcément des contradictions.

Le capital marchand en général - et le commerce des fourrures en particulier - qui n'était auparavant qu'un simple élément relevant de la préhistoire du capitalisme orienté vers le circuit économique français et existant «en marge» de la société canadienne, va perdre son autonomie en tant que procès de circulation pour être assujetti au procès de production. Il perdra ce statut d'appendice qui était sien sous le régime français. Il va devenir un agent du capital industriel et financier britannique. Par conséquent, il va pénétrer les autres modes et formes de production pour les dissoudre. Ce procès de dissolution et de destruction des modes et formes de production précapitalistes connaît, au Canada, un rythme particulièrement rapide, non sans rencontrer parfois de vives résistances. Mais il reste que ce procès qui a mis des siècles à se réaliser en Angleterre nécessitera moins d'un siècle au Canada ${ }^{25}$.

C'est dans l'évolution des structures régissant l'agriculture qu'on arrive le mieux à saisir les transformations que subissent, avec la Conquête, des structures et institutions en apparence demeurées les mêmes. On peut illustrer le phénomène en prenant pour exemple le régime seigneurial et, plus particulièrement, l'apparent renforcement de ses traits féodaux. L'examen de cette question permet également d'établir la rationalité économique sous-

24 Les spécialistes de l'histoire anglaise sont généralement d'accord pour faire débuter la révolution industrielle en Angleterre sensiblement à la même époque où se produit la Conquête (voir T.S. Ashton, La révolution industrielle, 1760-1830 (Paris, Plon, 1955); Christopher Hill, Reformation to industrial revolution (Penguin Books, 1969); Maurice Dobb, op. cit.).

Plusieurs auteurs signalent par ailleurs l'existence d'un décalage entre l'instance économique et les instances superstructurelles en ce sens que la dominance du MPC n'assure pas pour autant dans l'immédiat la domination politique et idéologique de la bourgeoisie. Voir à ce sujet Nicos Poulantzas, Pouvoir politique et classes sociales, Tome I (Paris, Petite collection Maspero, 1971): 179-184.

25 Cette compression du temps dans les situations coloniales en ce qui concerne la transformation des structures fait se poser la nécessité de développer des concepts et instruments d'analyse appropriés, ceux disponibles s'avérant parfois inadéquats. Des jalons ont été posés en ce sens par deux auteurs indiens qui introduisent le concept de «mode de production colonial». Voir Hamza Alavi, "India and the colonial mode of production», in Ralph Miliband et Saville (sous la direction de), Socialist Register (1975); J. Banasi, «For a theory of colonial modes of production", Economic and Political Weekly (Bombay), 8:2.

Pierre-Philippe Rey fait également usage d'un concept analogue dans Colonialisme, néo-colonialisme et transition au capitalisme (Paris, Maspero, 1971). 
jacente à l'alliance politique contractée après 1791 entre certains secteurs de la bourgeoisie marchande, l'administration déléguée et les éléments aristo-cléricaux.

L'historiographie libérale a vu dans la consolidation des traits féodaux du régime seigneurial un renforcement du féodalisme. C'est là prendre l'apparence pour la réalité et souscrire à une conception a-théorique de l'histoire. Car, au contraire, ainsi qu'il le sera démontré, ces faits témoignent d'une capitalisation de la rente foncière qui est à s'opérer et de la temporaire convergence des intérêts matériels de classes de la bourgeoisie marchande et des éléments aristo-cléricaux. Ce n'est que dans une phase ultérieure du procès d'articulation et de transition que la rente foncière deviendra néfaste et superflue pour le capitalisme. C'est pour cette raison qu'il y aura brisure au sein de l'alliance durant les années 1840 (c'est-à-dire une fois enrayée la menace issue du mouvement réformiste des années 1830), la bourgeoisie cherchant à affirmer son autonomie. Pour la même raison, il y aura disparition définitive du régime seigneurial par l'abolition de cette forme juridique de propriété en 1854. Pour le moment, cependant, on s'attachera plutôt à l'analyse de la première phase.

\section{B - La transition dans l'agriculture}

L'histoire de l'agriculture québécoise est soumise à l'influence de divers facteurs qui agissent simultanément, mais que, pour les fins de l'exposé, on doit considérer séparément. Les politiques impériales et la lutte des classes en métropole, les politiques coloniales en matière de distribution des terres, la lutte politique et les alliances de classes au sein de la colonie, voilà autant de facteurs que l'analyste se devra de retenir.

Ce qu'il faut arriver à montrer, c'est comment l'agriculture, organisée sur la base de rapports de production féodaux, est absorbée par le MPC suivant une séquence où elle est d'abord confrontée à la phase marchande dominante du développement du PMC puis où, par la suite, elle contribue à sécréter les structures mêmes d'où peut émerger le MPC de la phase industrielle.

À des fins strictement didactiques, l'exposé présentera d'abord les grandes tendances du développement, étant alors bien entendu que, dans leur action, les agents sociaux ne sont pas conscients de ces grandes tendances des lois du développement capitaliste. Dans un second temps, ces propos tendanciels seront nuancés lorsque cela s'avérera nécessaire. 
L'hypothèse retenue veut que la conjugaison spécifique des politiques métropolitaines et coloniales - en particulier, dans ce dernier cas, les pratiques adoptées par la bourgeoisie marchande quant à la gestion du territoire seigneurial qu'elle possédait - conduise à l'émergence de conditions favorables à l'éclosion du développement capitaliste de la phase industrielle. Or, le fait important est que certaines de ces politiques, notamment celles mises de l'avant par la bourgeoisie marchande locale, visaient précisément le contraire, c'est-à-dire qu'elles cherchaient à préserver le statu $q u o$, à savoir la dominance de l'échange marchand (ou procès de circulation).

Très tôt après la Conquête, les militaires puis les marchands anglais se portent acquéreurs de seigneuries ${ }^{26}$. A la différence de la plupart des seigneurs francophones - et ce, parce que plus rompus aux lois du capitalisme - ces marchands adoptent un mode de gestion de leurs seigneuries collant davantage à la réalité du marché27, c'est-à-dire: soumission aux lois de l'offre et de la demande, maximisation des profits, spéculation (on reporte à plus tard l'encaissement de profits que l'on estime devoir être plus considérables), notamment à partir du moment (1807) où le bois devient objet d'échange ${ }^{28}$. Bref, pour les marchands anglais, la seigneurie tend à être assimilée à une entreprise capitaliste. Tout ceci va se traduire par l'introduction d'un nouveau régime de gestion d'une fraction de plus en plus importante du territoire seigneurial: hausse des taux de cens et de rentes pour les nouvelles concessions, un meilleur ajustement des modalités de paiement de la rente (argent ou nature) à la conjoncture économique ${ }^{29}$, multiplication des réserves dans les

26 «Les marchands anglais, malgré la répulsion qu'inspirent de prime abord les rapports féodaux, n'hésitent pas à acquérir des seigneuries. Au cours de la première décennie qui suit la Conquête, leurs transactions portent sur 24 seigneuries, 3 parties de seigneuries et 10 arrière-fiefs. À partir de 1771 , les achats et ventes de fiefs se maintiennent à un rythme à peu près continu». Fernand Ouellet, «Le Régime seigneurial...», 167. Du même auteur, voir «Propriété seigneuriale...», 196 ss.

$27 \mathrm{Si}$ dans les lendemains immédiats de la Conquête, les seigneurs canadiensfrançais se montrent respectueux du droit coutumier français, plusieurs adopteront au cours du $19 \mathrm{e}$ siècle un mode de gestion qui manifeste une plus grande sensibilité aux fluctuations du marché, imitant en cela l'exemple des marchands anglais. On peut mentionner à cet égard le cas des seigneuries de Joly de Lotbinière (Voir Fernand Ouellet, Le BasCanada, 1791-1840 (Ottawa, Éditions de l'Université d'Ottawa, 1976), 229 ss.

28 Le bois devient une donnée essentielle de l'économie québécoise après 1807 . Bien que le gros de ce commerce soit tiré des cantons, on observe au 19e siècle un intérêt marqué de la part des seigneurs pour le bois. Ceux-ci ont de plus en plus tendance à se réserver le bois de construction (chêne et pin) ainsi que les emplacements pouvant servir à l'établissement de moulins à scies (Fernand Ouellet, Le Bas-Canada..., 232).

29 En fait, la forme de paiement varie au gré de la volonté du seigneur. Suivant les cycles de la conjoncture économique, il exigera paiement de la rente tantôt en nature, tantôt en argent. Quoi qu'il en soit, en raison de sa liaison étroite avec une conjoncture particulière, la modalité exigée est toujours celle qui est susceptible de faire le plus mal au censitaire. 
contrats, ralentissement et, souvent même, refus de concéder, rétablissement de droits tombés en désuétude ou jamais exercés, recours au droit de retrait et à la pratique du titre nouvel, pots-devin, etc. ${ }^{30}$.

Bien sûr, la logique d'une telle gestion conduit inévitablement $\mathrm{au}$ procès d'exclusion de l'accès à la propriété foncière. Mais la bourgeoisie marchande ne le voit pas ainsi, et tel n'est pas son objectif, bien au contraire. Pour elle, l'objectif est double. Elle a, d'une part, intérêt à voir se développer une agriculture commerciale pour pouvoir en tirer profit, pour autant que ce type d'agriculture soit confiné à la satisfaction d'une demande extérieure. Étant donné les structures de la propriété foncière, la bourgeoisie marchande peut compter sur deux types de revenus tirés de l'agriculture: les redevances et exactions seigneuriales et le prélèvement d'une quote-part (profit) sur la commercialisation des produits transigés avec la métropole. La présence de ces marchands dans le secteur agricole peut, en outre, s'expliquer par la dominance du MPC de la phase marchande et les besoins spécifiques de la nouvelle métropole qui laissent entrevoir l'élargissement de la liste des produits d'échange, et par l'expérience anglaise qui suggère la hausse constante de la valeur de la propriété du sol. À cet égard donc, il ne faudrait pas négliger l'attirance qu'exerce la pure spéculation pour justifier la présence marchande dans l'agriculture bas-canadienne.

D'autre part, cette classe cherche à affermir son hégémonie dans la colonie par la préservation du statu quo économique, c'està-dire le maintien de structures économiques fondées sur la circulation et non sur la production de biens. En d'autres termes, il est dans l'intérêt de la bourgeoisie marchande de freiner le développement de l'industrie, c'est-à-dire d'un secteur manufacturier élargi.

Ce qu'il faut bien voir, c'est que ces objectifs sont, à long terme, contradictoires, suivant les lois du développement du capitalisme. L'instrument de réalisation de chacun conduit ultimement au procès de dépossession de la paysannerie. En effet, la réalisation de chacun de ces objectifs (commercialisation limitée et frein au développement industriel) s'alimente au même train de mesures: maintien de taux de cens et de rentes élevés pour les nouvelles concessions, ajustement des modalités de paiement de la rente à la conjoncture économique, restriction de l'accès à la propriété foncière, etc. La commercialisation de l'agriculture passe, entre autres, par la production de surplus et, par conséquent, par la

30 Fernand Ouellet, «Le régime seigneurial...», 167, 170, 171. Voir également son Histoire économique et sociale du Québec, 1760-1850 (Montréal, Fides, 1971) 2: 351 ss. 
capacité de forcer l'agriculteur à se présenter sur le marché. Les taux élevés des redevances, tout comme l'exigence des paiements en argent, agiront en ce sens. Cependant, il y a un seuil critique à ne pas dépasser car, au-delà de ce seuil, l'agriculteur, incapable de soutenir des charges trop onéreuses, est contraint d'abandonner la terre, ce qui contribue à faire grossir les rangs de la masse des «travailleurs libres» et, partant, favorise l'avènement de la phase industrielle du développement du MPC.

Par ailleurs, le maintien de l'hégémonie économique de la bourgeoisie marchande par le freinage du développement d'un secteur manufacturier suppose que l'on réussisse à limiter l'accès à la propriété foncière pour éviter la production des surplus nécessaires au ravitaillement de la main-d'oeuvre du secteur industriel. À court terme l'instrument est bien ajusté à l'objectif. À plus long terme, cependant, une politique de restriction de l'accès à la propriété foncière produit précisément le contraire de l'effet désiré, puisqu'elle contribue à la création d'une réserve de «travailleurs libres ${ }^{31}$. Bref, les instruments mis en oeuvre conduisent inévitablement au procès de dépossession de la paysannerie, prérequis au développement de la phase industrielle du MPC.

Il ne faudrait pas, pour autant, laisser l'impression que la bourgeoisie marchande est seule responsable de la mise en place de structures favorables à l'émergence du capitalisme industriel, ni qu'elle - ou toute autre classe - a une conscience claire des lois historiques du développement capitaliste et qu'elle y modèle ses comportements, ni faire apparaître comme étant clairement défini dans son esprit le rapport objectifs-moyens. Ce serait là conférer un aspect trop volontaire à l'action de cette classe et, du même coup, minimiser son désir, plus banal, de vouloir tout simplement s'enrichir et spéculer. Car outre les motifs à caractère nonéconomique qui ont été à l'origine de l'incursion d'une fraction de la bourgeoisie marchande du côté de la propriété foncière, ses objectifs immédiats seront définis par rapport aux structures existantes qui offrent la possibilité de réaliser des bénéfices intéressants, ainsi qu'en réponse à certains mouvements conjoncturels. D'ailleurs, à ce chapitre, le désintéressement de la bourgeoisie marchande vis-à-vis du régime seigneurial - elle ira jusqu'à en récla-

31 Déjà en 1830 , ce réservoir de main-d'oeuvre a commencé à se constituer par suite de l'explosion démographique et des pressions que celle-ci engendre sur le territoire seigneurial. Le nombre des non-propriétaires s'accroît considérablement en milieu rural. L'infrastructure industrielle est trop peu développée pour pouvoir absorber une partie importante de cette main-d'oeuvre excédentaire. Ce sont les entreprises forestières qui seront, à cette époque, les principales bénéficiaires de la constitution de ce prolétariat rural. Voir Jean Hamelin et Fernand Ouellet, «Les rendements agricoles dans les seigneuries et les cantons du Québec: 1700-1850», in Claude Galarneau et Elzéar Lavoie (sous la direction de), op. cit., 106-107. 
mer l'abolition ${ }^{32}$ - coïncidera avec la fin de la production de surplus commercialisables destinés à l'exportation par suite, notamment, de l'appauvrissement des sols et du non-déploiement des innovations technologiques disponibles ${ }^{33}$. Désormais, l'agriculteur s'emploie à la seule satisfaction de ses besoins immédiats. Le secteur agricole perd alors beaucoup de son intérêt pour la bourgeoisie marchande.

On se doit également de dissiper une autre fausse impression qui pourrait naître des propos tenus plus haut. Il ne faudrait pas croire, en effet, que la bourgeoisie marchande réussisse à contenir tout développement manufacturier. Au contraire, même si elle ne retient pas ici l'attention parce qu'elle se situe hors du champ du présent propos, il existe, durant les années 1830 et 1840 , toute une couche de petits entrepreneurs propriétaires de manufactures dont le nombre va croissant au fur et à mesure où la structure économique de la colonie se diversifie ${ }^{34}$. Ils sont très actifs sur la scène politique locale, même si l'expansion de leur secteur est inhibée par les lois métropolitaines restrictives de l'activité manufacturière au sein des colonies ainsi que par certaines dispositions du régime seigneurial, notamment celles concernant les lods et ventes ${ }^{35}$. Ils font d'ailleurs des revendications visant précisément à faire lever ces restrictions, revendications qui recevront l'appui d'une fraction du Parti patriote $^{36}$.

Dans l'immédiat, pour réaliser ses objectifs, la bourgeoisie marchande doit se gagner l'appui des autres catégories de propriétaires fonciers, notamment celui de la classe seigneuriale traditionnelle (canadienne-française) ${ }^{37}$. Une fraction importante de cette

32 La bourgeoisie marchande est quelque peu divisée sur cette question. La fraction de la bourgeoisie marchande qui a amorcé une diversification de ses activités économiques - notamment ceux qui délaissent la circulation au profit des secteurs productifs - est plus sensibilisée à l'urgence de se départir d'un régime de propriété qui inhibe le développement du capitalisme que cette autre fraction engagée presque exclusivement dans l'échange marchand et pour qui les bénéfices tirés d'une exploitation seigneuriale l'emportent sur les inconvénients causés par cette forme de propriété.

33 Fernand Ouellet, Le Bas-Canada..., 232 ss; «Le régime seigneurial...», 169 ss. 1972), 45-49.

34 Stanley B. Ryerson, Le capitalisme et la Confédération (Montréal, Parti Pris,

35 Il s'agit là d'un impôt de mutation qui permet au seigneur de s'approprier un douzième du prix de vente. Ce droit s'avère particulièrement onéreux en milieu urbain où les manufactures sont érigées sur des terres concédées.

36 La presse patriote dénonce le gouvernement britannique «qui, non seulement se refuse d'encourager les manufactures parmi nous, mais qui empêche leur établissement» (La Minerve, 18 juillet 1836). Les Canadiens doivent prendre «les moyens de se rendre indépendants de l'Angleterre en fabriquant chez eux le papier, le cuir, les draps, les toiles et autres articles d'un usage continuel et nécessaire» (La Minerve, 14 avril 1836).

37 Déjà, l'introduction de la tenure en franc et commun soccage (1791), ainsi que la cession de vastes territoires de la Couronne à la British American Land Company, en 1832, assurent le maintien de prix élevés pour l'accès à la propriété foncière en ce qui a trait aux terres situées hors du territoire seigneurial. Il reste dès lors à convaincre les seigneurs canadiens-français traditionnels de pratiquer un mode de gestion qui ira dans le même sens. 
dernière en viendra à calquer sa gestion du territoire seigneurial sur le modèle mis de l'avant par les seigneurs-commerçants anglais parce qu'elle y trouve la satisfaction de ses intérêts matériels immédiats qui sont, par ailleurs, très différents de ceux de la bourgeoisie marchande. La Conquête a contribué à accentuer la féodalisation de la classe seigneuriale traditionnelle en la coupant des revenus qu'elle tirait du commerce sous le régime français. Si bien qu'à quelques exceptions près (Barthélemy Joliette par exemple), les seigneurs canadiens-français constituent une classe de propriétaires fonciers féodaux. De sorte que lorsque cette classe consent à s'éloigner des prescriptions du droit coutumier français en recourant aux stratagèmes mis de l'avant par les seigneurs-commerçants anglais $^{38}$, il faut y voir, de son point de vue, un renforcement de l'extorsion féodale et non la ponction d'une rente foncière de type capitaliste. Or, il arrive que ce besoin d'accroissement de la rente féodale ressenti par la classe seigneuriale traditionnelle rencontre les intérêts immédiats de la bourgeoisie marchande capitaliste, car elle l'assure d'un ralentissement dans la distribution des terres, les coûts d'acquisition d'un titre de propriété devenant trop onéreux pour beaucoup d'aspirants à ce statut. D'où l'alliance politique contractée par ces deux classes.

La symétrie apparente des comportements répond cependant à des impératifs différents. On connaît les motivations des seigneurs-commerçants anglais. Par ailleurs, pour les seigneurs traditionnels, la politique de hausse des taux de cens et de rentes appliquée sur les territoires qu'ils contrôlent, traduit une importante modification de la position économique de cette classe par suite de la Conquête. Bon nombre de ces seigneurs se voient, en effet, amputés d'une importante source de leur revenu tel qu'il était constitué sous le régime français (ravitaillement de l'armée, occupation de certaines charges administratives et, dans certains cas, participation au secteur d'échange avec la métropole) ${ }^{39}$. Coupée des anciens circuits commerciaux métropolitains - tant en ce qui concerne l'accès au crédit que l'écoulement des stocks - et écartée d'un accès direct aux nouveaux à la faveur de la nouvelle bourgeoisie marchande anglaise, la classe seigneuriale traditionnelle doit combler son manque à gagner en accroissant les revenus tirés de l'exploitation des seigneuries.

Les seigneurs recrutés au sein de la bourgeoisie marchande anglaise se retrouvent, pour leur part, dans la position qui était celle de beaucoup de seigneurs canadiens-français sous le régime français, c'est-à-dire tirant l'essentiel de leurs revenus d'une acti-

38 Fernand Ouellet, «Libéré ou exploité...», 348.

39 Fernand Ouellet, "Le régime seigneurial...», 168. 
vité commerciale. Position améliorée, cependant, du fait de la plus grande ampleur de l'échange commercial avec la nouvelle métropole et de l'élargissement du commerce interne après 1807, favorisant, on peut le supposer, la jouissance de revenus plus élevés tirés du commerce. En outre, la plus grande proximité d'avec le MPC achevé leur dictera des comportements quelque peu différents de ceux adoptés par les seigneurs sous la Nouvelle-France. Ainsi, les activités de pure spéculation occupent une place plus importante. Cela est dû à une plus grande imminence de l'encaissement des profits anticipés.

Par ce qui précède, on constate que l'alliance politique contractée entre 1791 et les années 1840 par la bourgeoisie marchande et les éléments aristo-cléricaux repose sur certaines bases matérielles. Chaque classe y trouve son compte. La première assure la dominance de la circulation sur la production des biens. La seconde accroît des revenus, tout en contribuant, indirectement, à la réalisation des objectifs visés par la bourgeoisie marchande.

Cependant, au plan économique, cette alliance ne saurait être que transitoire; l'expression de la rencontre momentanée d'intérêts. Car, à plus long terme, les dispositions prises en ce qui a trait à la gestion du sol, quel que soit le mode de tenure, produiront des effets qui seront en contradiction avec les intérêts de la bourgeoisie marchande, de même qu'avec les objectifs poursuivis par la métropole. Elles auront, en effet, pour conséquence de hâter la dépossession d'une partie importante de la paysannerie, créant ainsi des conditions favorables au passage à la phase industrielle du développement du MPC.

En bref, l'orientation prise par l'agriculture sous l'impulsion de la bourgeoisie marchande porte les germes du propre dépassement de cette classe en ce qu'elle mène, à plus ou moins long terme, à l'émergence du capitalisme de la phase industrielle. Telle n'est certes pas la volonté de la bourgeoisie marchande (c'est-àdire engendrer les instruments de son propre dépassement), mais tel est précisément le résultat, lorsque les diverses dispositions sont appréciées à la lumière des lois de l'histoire en ce qui concerne les étapes régissant le passage du féodalisme au capitalisme et, plus proprement, à l'intérieur du MPC lui-même, le passage de la phase marchande à la phase industrielle. Comment opère ce procès de transition déclenché par la bourgeoisie marchande avec la complicité de la classe seigneuriale traditionnelle? ${ }^{40}$

40 Si l'accent est ici placé sur le rôle de la bourgeoisie marchande dans le procès de transition, d'autres facteurs qui concourent à accélérer cette transition sont également à l'oeuvre. On peut mentionner à cet égard les effets de l'explosion démographique que connaît le Québec de l'époque de même que les fluctuations de la politique coloniale anglaise (infra, 89 ss). 
Si la politique poursuivie par la bourgeoisie marchande semble favoriser ses intérêts économiques par un aspect, elle entre également en contradition avec ceux-ci par un autre aspect. En adoptant, pour le territoire seigneurial qu'elle contrôle, un mode de gestion plus proche du capitalisme, la bourgeoisie marchande force éventuellement l'agriculteur à se présenter sur le marché ou à quitter la terre. Car, d'une part, le ralentissement du rythme des concessions en territoire seigneurial et le maintien de prix élevés pour les terres exploitables en franc et commun soccage servent assez bien une politique de restriction de la production commercialisable sur le marché local, dont l'absence retarde le développement du procès d'industrialisation. Mais, d'autre part, les mouvements à la hausse des taux de cens et de rentes pour les nouvelles concessions - ainsi que les diverses pratiques qui conduisent à des résultats analogues pour d'anciennes concessions - de même que l'incertitude qui plane à chaque année sur le censitaire quant aux modalités de paiement de la rente, contribuent, en revanche, à la création de conditions favorables au procès d'industrialisation. De sorte qu'au total, cette politique crée, ultimement, la nécessité d'un revenu d'argent, lequel ne peut être obtenu que par l'intégration de l'agriculteur à une économie de marché. Ce dernier, pour faire face à ses obligations, est contraint d'augmenter sa production bien au-delà du seuil de la subsistance d'autrefois. Il lui faut générer des surplus commercialisables. Or la bourgeoisie marchande ne peut avoir l'assurance que ces surplus seront toujours écoulés sur les marchés extérieurs. Ainsi, rapidement, les lois du capital auront pénétré l'agriculture à compter du tournant du XVIIIe siècle.

Ces développements témoignent bien du caractère de «période de transition» engendrée par la Conquête. On parle de "transition", parce que l'on se trouve désormais dans l'impossibilité d'assurer intacte la reproduction des rapports de production dominés jusque-là par des traits féodaux. Les contradictions foisonnent, se multiplient, de sorte que les rapports de production sont constamment modifiés dans leur reproduction. Il en sera ainsi jusqu'à ce que le MPC de la phase industrielle ne devienne dominant au niveau des rapports de production, ce qui sera fait durant le dernier tiers du XIXe siècle. Cependant, les mécanismes y conduisant sont d'ores et déjà à l'oeuvre.

Ainsi, à compter de la fin des années 1820 , sous le poids de l'effet des diverses mesures évoquées, une fraction importante de la paysannerie quitte la terre, soit parce qu'elle est acculée à la faillite, c'est-a-dire incapable de rencontrer les paiements exigés et les redevances à acquitter; soit parce qu'elle est dans l'impossibilité d'assumer le coût d'achat d'une terre nouvelle, quel que soit le 
mode de tenure. De même, le mouvement spéculatif qui s'empare de nombreux seigneurs - notamment à partir du moment où le bois devient, dans l'économie canadienne, un produit commercialisable - et qui se traduit par des refus de concéder, contribue à faire augmenter le nombre des «sans terres»" ${ }^{41}$. De sorte qu'à plus ou moins brève échéance, divers moyens, non destinés à cette fin spécifique, ont néanmoins contribué à l'expulsion d'une partie importante de la paysannerie.

Or les paysans dépossédés ne servent plus la cause de la bourgeoisie marchande ni celle de la classe seigneuriale traditionnelle, puisqu'ils sont non-payeurs de cens et de rentes et non-générateurs de profits appropriés par les commerçants dans l'agriculture commerciale. Mais il constituent, par ailleurs, un des deux ingrédients indispensables au développement du capitalisme de la phase industrielle, à savoir une masse de «travailleurs libres». Or nul doute que la phase industrielle aurait démarré plus rapidement au Canada si ce n'avait été des mesures restrictives impériales touchant le commerce extra-colonial, l'établissement de manufactures et la production de certaines catégories de biens. À cet égard, la bourgeoisie métropolitaine a précisément tout intérêt à prolonger le plus longtemps possible la phase marchande du développement capitaliste dans la colonie puisqu'elle s'avère une source d'accumulation de capital, lequel est réinvesti dans les industries anglaises.

\section{C-Le rôle de la métropole dans le procès de transition et d'articulation}

La dépossession de la paysannerie ne produira pas ses effets de manière aussi mécanique et immédiate que pourraient le laisser croire les paragraphes précédents. En d'autres termes, la présence d'une masse de «travailleurs libres» ne débouche pas nécessairement sur le passage immédiat à la phase industrielle. C'est ici que doit être introduite toute la dimension coloniale qui vient médiatiser le procès d'expulsion de la paysannerie. Ce procès se déroule, en effet, dans le cadre d'une domination exercée par la métropole pour laquelle la colonie existe d'abord en fonction de l'assouvissement de ses propres besoins, en l'occurrence pour s'approvisionner en matières premières et produits primaires et ouvrir une source de débouchés à ses produits manufacturés, ce qui l'amène à prendre

41 Les refus de concessions touchent plus particulièrement les terres dites en «bois debout», c'est-à-dire non défrichées, et partant donc, les plus susceptibles de faire l'objet d'une concession puisque faisant partie des censives non-concédées d'un territoire seigneurial. 
des mesures visant à freiner la liberté du commerce dans la colonie et à y restreindre le développement des manufactures.

Or jusqu'à présent, l'examen du procès de transformation des structures socio-économiques de la colonie et, plus particulièrement, de l'absorption de l'agriculture par le MPC, a été circonscrit au rôle des facteurs internes. Il faut maintenant toucher au rôle de la métropole. Ce procès de transformation est, en effet, scandé par deux ordres de phénomènes.

Le premier est extérieur à la colonie et échappe totalement à son contrôle. Il s'agit des politiques impériales. Le second a déjà été abordé. Il renvoie au développement des forces productives et des rapports de production dans la colonie, lequel est, dans une certaine mesure, conditionné par les diktats de la métropole, tout en étant doté d'une dynamique propre fondée sur les conditions réelles d'existence dans la colonie. Cette double sujétion du procès de transformation conduit à des contradictions. Certaines d'entre elles sont déjà connues. On verra plus loin que d'autres sont attribuables aux agissements de la métropole.

En incorporant cette dernière à l'analyse, il faut cependant se garder de «figer» son évolution. L'aventure coloniale anglaise sera, en effet, soumise aux divers moments de l'expression de la lutte des classes en Angleterre, de même qu'à l'évolution du développement de ses propres forces productives. D'où les contradictions que l'on pourra observer au fil des années dans le contenu de diverses politiques touchant le Canada.

Toute analyse portant sur une plus ou moins longue période se doit donc de prêter une attention particulière aux fluctuations de la lutte des classes en métropole. Les rapports politiques et les alliances se transforment durant tout ce temps et influent sur les politiques adoptées à l'endroit de la colonie ${ }^{42}$. De sorte que la lutte politique de classes en métropole constitue une des composantes essentielles de l'explication de certaines contradictions qui seront relevées plus loin. Ces contradictions ajoutent à la complexité de l'analyse du double procès de transition qui a cours au Canada

42 En somme, il faudrait posséder une certaine expertise sur l'histoire de l'Angleterre pour éclairer plus justement la transformation des structures socio-économiques canadiennes dans la mesure où le contexte de domination coloniale constitue un paramètre essentiel. L'auteur n'a aucune prétention en ce sens à ce stade-ci de ses recherches. Par ailleurs, à cet égard, les travaux de Peter Burroughs et de Helen Taft-Manning sont fort utiles. Du premier, voir, The Colonial Reformers and Canada, 1830-1849 (Toronto, McClelland et Stewart, 1969); British Attitudes towards Canada, 1822-1849 (Scarborough, PrenticeHall, 1971). De Taft-Manning, voir "The colonial policy of the Whig Ministers, 18301837», Canadian Historical Review, XXXIII, 3 (septembre 1952): 203-236; XXXIII, 4, (décembre 1952): 341-368. Voir également les travaux d'historiens mentionnés aux notes 4 et 24. Consulter aussi Eric-J. Hobsbawn, L'ère des révolutions (Paris, Fayard, 1967); Industry and Empire (Harmondsworth, Penguin Books, 1969). 
entre 1760 et 1850 , soit le passage de la dominance de rapports de production de type féodal à des rapports capitalistes, passage qui s'amorce dans l'agriculture avec la Conquête, puis, à compter de la fin des années 1840, le passage de la phase marchande à la phase industrielle du MPC.

Situons dans ses grandes lignes l'évolution de la politique coloniale anglaise à l'égard du Canada. Dans un premier temps, elle assure l'hégémonie du capital marchand et tend à retarder l'apparition du capital industriel en limitant l'expansion du secteur productif. De fait, le seul intérêt de la métropole réside dans un monopole de l'échange. Diverses lois impériales sont mises à contribution: lois de navigation restreignant la liberté de commerce; lois prohibant l'établissement de manufactures pour la production de certaines catégories de biens; lois restreignant l'immigration de main-d'oeuvre spécialisée; maintien de la tenure seigneuriale, etc.

Puis à compter de la fin des années 1820 surtout, en agissant par une série de mesures législatives sur les régimes de propriétés foncières et sur les conditions d'exercice de l'échange commercial, on neutralise l'effet des politiques antérieures et, ce faisant, on ouvre la voie aux transformations qui conduiront à la phase industrielle du développement du MPC. Ces diverses lois émanent tantôt du gouverment impérial lui-même et tantôt du gouvernement colonial avec l'assentiment de Londres. Parmi ces mesures législatives, mentionnons l'introduction d'une tenure parallèle, celle en franc et commun soccage (1791); la vente aux enchères des terres de la Couronne au lieu de la cession gratuite qui avait prévalu jusque-là (1826); le gel définitif de l'expansion du territoire seigneurial (1831), bien que dans la pratique un tel mouvement se soit amorcé dès 1791; la vente de territoires de la Couronne situés dans les Cantons de l'Est à la British American Land Co. (1832), entreprise capitaliste qui entend fixer ses prix en fonction des lois de l'offre et de la demande; les diverses lois $(1822,1825,1826,1827$, 1831) menant éventuellement au libre-échange (1846) et contribuant ainsi à affaiblir à la fois les agriculteurs commerciaux et la bourgeoisie marchande coupés des marchés protégés ${ }^{43}$.

Il faudrait scruter sérieusement l'histoire de la politique intérieure anglaise pour établir la volonté exacte de l'Angleterre dans

43 Pour plus de détails sur toutes ces questions, voir Maurice Séguin, op. cit., 177 203; D.G. Creighton, The empire of the St. Lawrence (Toronto, MacMillan, 1970), 231 ss; Adam Shortt, "General economic history, 1763-1841», in Adam Shortt et Arthur G. Doughty (sous la direction de), Canada and its provinces, 4, section II, British Dominion, 2e partie (Toronto, Glasgow, Brook and Co., 1914): 569-575; Jacques Boucher, "Les aspects économiques de la tenure seigneuriale au Canada (1760-1854)», in P. Solomon et autres, Recherches d'histoire économique (Paris, PUF, 1964), 149-209. 
ce dossier, de même que pour déterminer si elle avait une idée précise des conséquences que ce train de mesures allait produire. En regard de cette dernière question, on peut être enclin à répondre par l'affirmative si l'on tient compte que la métropole était forte de sa propre expérience en la matière et que, par ailleurs, certains textes de Wakefield (alors secrétaire aux colonies) font état de la relation existant entre la facilité d'accès à la petite propriété foncière et la rareté et la cherté de la main-d'oeuvre ${ }^{44}$.

Il ne faudrait cependant pas exagérer en ce sens puisque, pour prendre un exemple précis, la vente de territoires de la Couronne à une société privée n'a rien à voir avec les propos tenus par Wakefield. Elle correspond à des besoins précis de la métropole et des administrateurs locaux, à savoir: générer des revenus additionnels pour réduire les frais d'entretien de la colonie par la métropole et assurer au Conseil exécutif des rentrées de fonds qui soient soustraites au contrôle de l'Assemblée législative. Cet exemple laisse par ailleurs entrevoir les contradictions au sein de la politique impériale, puisque l'instrument choisi pour obtenir un gain immédiat compromet à plus long terme les intérêts de la métropole, notamment son désir de limiter le développement du secteur productif ${ }^{45}$.

Quoi qu'il en soit, toutes ces décisions ont pour effet de rendre plus difficile l'accès à la propriété foncière. Le gel du territoire seigneurial favorise la spéculation qui a déjà cours, incite au refus de concéder ainsi qu'à la multiplication des réserves dans les contrats et, finalement, encourage le maintien de taux de cens et de rentes élevés. La remise à l'entreprise privée d'une vaste portion des terres publiques constitue un autre dur coup aux espoirs de

44 Voir les propos cités in Stanley B. Ryerson, op. cit., 41-42.

45 Car bien sûr, en multipliant les difficultés d'accès à la petite propriété foncière la vente de territoires de la Couronne à une société privée va en ce sens - on contribue à créer des surplus de main-d'oeuvre qui pourraient être absorbés par le développement d'un secteur productif. De plus, en abolissant les tarifs préférentiels consentis aux colonies, il devient plus difficile de justifier le maintien du contrôle exclusif exercé par la métropole sur les tarifs imposés aux produits importés dans la colonie. Cette dernière obtiendra son autonomie fiscale en 1849 et pourra se doter d'une politique tarifaire - notamment en regard des importations - qui favorisera l'expansion de l'industrie manufacturière locale.

Dès le début des années 1840, la bourgeoisie marchande liée au marché impérial s'inquiétant des tendances libre-échangistes en Grande-Bretagne avait prévu ce qui allait survenir après 1846 . Faisant valoir la nécessité de maintenir des tarifs préférentiels généreux sur le blé et le bois, elle met en garde la métropole en ces termes:

"Unless the colonies were encouraged to devote themselves exclusively to the production of agricultural produce and of other raw materials, they would undoubtedly turn their attention to manufacturing before long, and attempt to pass laws for its protection from outside competition» (Mémoire de la North American Committee of the Colonial Association cité in Adam Shortt, "Economic history, 1840-1867», in Adam Shortt et Arthur G. Doughty (sous la direction de), Canada and its provinces, 5, section III, United Canada (Toronto, Glasgow, Brook and Co., 1914): 196. 
ceux qui aspirent à la propriété. Car dans une société où le revenu d'argent est rare parmi les classes populaires, l'accumulation nécessaire pour défrayer le coût d'achat d'un titre de propriété est très difficile.

Ainsi, les fluctuations des politiques de la métropole constituent une source de contradictions qui viennent s'ajouter à celles qui trouvent leurs origines dans la colonie. La volonté initiale de l'Angleterre de prévenir le développement d'un secteur productif est neutralisée, plus tard, par ses mesures de réglementation de la propriété foncière. Celles-ci viennent se conjuguer aux pratiques suivies par les classes possédantes coloniales en matière de gestion du sol, pratiques elles-mêmes empreintes de contradictions, ainsi qu'on l'a démontré plus haut.

On voit bien la complexité du problème. Toute situation coloniale sécrète de nombreuses contradictions puisqu'elle répond, en quelque sorte, à deux dynamiques qui ne sont pas forcément synchronisées, celle de la métropole et celle de la colonie. C'est pourquoi, dans une telle situation, le contrôle de la métropole n'est jamais absolu. Celui-ci se heurte à une dynamique propre à la colonie. Cette dynamique, de même qu'une certaine marge d'autonomie, lui sont conférées par le développement spécifique des forces productives et des rapports de production dans la colonie. Il y a donc possibilité de décalage entre des mesures prises à distance, hors du terrain, et leur application sur un terrain concret, dynamique et mouvant. Ce faisant, les rapports métropole-colonie constituent une seconde source de contradictions venant s'ajouter à celles inhérentes à l'évolution de toute formation sociale.

Il apparaît donc, en dernière analyse, que si on peut les freiner, les déformer dans l'immédiat, à long terme, on ne peut contraindre par voie législative les tendances historiques qui président au développement d'un mode de production.

L'aboutissement de ces contradictions, combiné à l'accession définitive au pouvoir, dans la métropole, des représentants d'une bourgeoisie industrielle récusant le protectionnisme et vouée à la promotion d'une politique de libre-échange, modifiera substantiellement la structure économique canadienne, conduisant ultimement à la levée des obstacles inhibiteurs du développement de la phase industrielle du MPC au Canada.

L'abolition des tarifs préférentiels sur le blé (Corn Laws de 1846) et sur les produits de la forêt (1851) produira dans la colonie des conséquences menant simultanément à une profonde transformation des structures économiques canadiennes, ainsi qu'à des mutations substantielles au sein de la structure sociale coloniale; 
les principales données sur ces deux plans sont l'effrondrement soudain mais temporaire de la bourgeoisie marchande de Montréal, la perte de son hégémonie économique, l'apparition de nouvelles classes, la consolidation de la position des petits entrepreneurs manufacturiers, la lutte victorieuse pour faire lever les entraves à l'élargissement de la liste des partenaires commerciaux et à la diversification du secteur productif et, bien sûr, l'émancipation politique par l'application du principe de la responsabilité ministérielle ${ }^{46}$.

De sorte qu'au début des années 1850 , c'est-à-dire lorsque les conditions d'émergence de la phase industrielle se seront suffisamment développées, on assistera à une intensification du mouvement en vue de l'abolition du régime seigneurial. Cet assaut concerté atteste dans quelle mesure la transition s'est rapprochée de son point d'aboutissement et ce, tout particulièrement en milieu urbain $^{47}$. Paysans exploités, membres de la bourgeoisie industrielle naissante, certains éléments de la bourgeoisie marchande ayant diversifié leurs activités économiques, clameront - souvent pour des raisons fort différentes - leur opposition au maintien de ce régime de propriété foncière. Pour les industriels et une partie de la bourgeoisie marchande, la tenure seigneuriale - en particulier sur le territoire urbain - contrevient à la nécessité capitaliste de la propriété absolue du sol sur lequel on entend ériger des manufactures, des établissements commerciaux ou des entrepôts. À cet égard, les droits de lods et ventes et de retrait laissent planer sur les entreprises capitalistes un climat d'insécurité inacceptable ${ }^{48}$.

\section{Conclusion}

Le recours aux notions de transition et d'articulation de modes de production, en ce qu'il force à intégrer davantage la métropole à l'analyse, contribue à mieux éclairer un fait de l'his-

46 Pour une analyse détaillée de toutes ces questions, voir Gérald Bernier, Développement politique, dépendance et analyse historique. Un champ d'application: la société québécoise de 1837 à 1867, thèse de Ph.D. en science politique (Université Laval, 1977), chap. 3 .

47 Plusieurs travaux récents sur Montréal contribuent à une meilleure connaissance de l'implantation du MPC achevé au Québec, illustrent le rôle d'avant-garde joué par Montréal à cet égard et permettent de comprendre la répugnance qu'éprouvent les milieux urbains pour la tenure seigneuriale en cette ère de transition. Voir Robert Tremblay, «La formation matérielle de la classe ouvrière à Montréal entre 1790 et 1830", Revue d'histoire de l'Amérique française, 33, 1, (juin 1979): 41-50; Joanne Burgess, "L'industrie de la chaussure à Montréal: 1840-1870 - Le passage de l'artisanat à la fabrique», in idem, 31, 2 (septembre 1977): 187-210; Paul-André Linteau et Jean-Claude Robert, «Propriété foncière et société à Montréal: une hypothèse», in idem, 28, 1 (juin 1974): 45-65; Jean-Claude Robert, "Les notables de Montréal au XIXe siècle», Histoire sociale, VIII, 15 (mai 1979): 54-76. 353-357.

48 Voir Fernand Ouellet, «Le régime seigneurial...», 169; Histoire économique..., 
toire québécoise qui est trop souvent négligé. A savoir, la mise en place des mécanismes conduisant à l'expulsion de la paysannerie avant même que ne se soit développée la capacité d'absorption de ce surplus. Ce fait est, en grande partie, attribuable à la dimension coloniale, et non à la seule action des classes locales sur le développement des forces productives. Les contradictions de la politique coloniale métropolitaine issues de l'adoption de mesures conduisant à l'expulsion de la paysannerie et d'autres visant à contenir le développement du secteur manufacturier, rendent compte du retard accusé par la capacité d'absorption. Il s'ensuit que la première génération de prolétaires québécois se constitue outrefrontière. Les paysans québécois dépossédés se retrouvent, en effet, dans les villes industrielles de la Nouvelle-Angleterre, fournissant une main-d'oeuvre bon marché aux industries textiles, contribuant ainsi à l'accélération du développement industriel du nord-est des États-Unis. Un tel fait n'est pas sans analogie avec la situation contemporaine rencontrée dans de nombreux pays dits en voie de développement (par exemple, les travailleurs NordsAfricains en France, les Pakistanais et les Hindous en Angleterre, les Mexicains aux États-Unis, etc.).

Il convient de signaler en terminant que tout le phénomène de l'émigration massive de Canadiens français vers les États-Unis durant le $\mathrm{XIX}^{\mathrm{e}}$ siècle constitue un événement dont les conséquences n'ont pas suffisamment été évaluées par les historiens. Si les aspects quantitatifs et les conditions d'établissement dans la patrie d'adoption sont assez bien connus, en revanche, on ignore tout des conséquences de ce mouvement migratoire sur le système politique québécois. Entre autres, il serait important d'évaluer sa fonction de substitut à la lutte des classes à une époque où précisément la transition vers le capitalisme industriel produit ses effets de désorganisation sociale les plus aigus. Une interrogation sur la relation possible entre le mouvement migratoire et l'absence de radicalisme politique durant le dernier quart du XIX ${ }^{\mathrm{e}}$ siècle - notamment au niveau des partis politiques - serait également de mise. 